\title{
OSCILLATION THEOREMS FOR THE REAL, SELF-ADJOINT LINEAR SYSTEM OF THE SECOND ORDER*
}

BY

\section{H. J. ETTLINGER}

\section{INTRODUCTION}

It is the object of this paper to determine the number of oscillations of a linear combination of the form (2) for the systems (3) and (4). From these results, an oscillation theorem for the solution $u_{p}(x)$, corresponding to the $p$ th characteristic number of (4), is obtained.

Given the second order self-adjoint linear differential equation

$$
\frac{d}{d x}\left[K(x, \lambda) \frac{d u}{d x}\right]-G(x, \lambda) u=0
$$

and two linear combinations of a solution which does not vanish identically and its first derivatịe,

(2) $L_{i}[u(x, \lambda)]=\alpha_{i}(x, \lambda) u(x, \lambda)-\beta_{i}(x, \lambda) K(x, \lambda) u_{x}(x, \lambda) \quad(i=1,2)$,

we shall impose the following conditions and shall assume that they are satis. fied throughout this paper:

I. $K(x, \lambda), G(x, \lambda), \alpha_{i}(x, \lambda), \beta_{i}(x, \lambda), \alpha_{i x}(x, \lambda), \beta_{i x}(x, \lambda) \dagger$ are continuous, real functions of $x$ in the interval

$$
(a \leqq x \leqq b)
$$

and for all real values of $\lambda$ in the interval

$$
\left(\mathfrak{L}_{1}<\lambda<\mathscr{L}_{2}\right) \text {. }
$$

II. $K(x, \lambda)$ is positive everywhere in $(X, \Lambda)$ and

$$
\left|\alpha_{i}\right|+\left|\beta_{i}\right|>0
$$

in $(\boldsymbol{X}, \boldsymbol{\Lambda})$.

III. For each value of $x$ in $(X), K$ and $G$ decrease (or do not increase)

* Presented to the Society, Sept. 6, 1917.

$\dagger f_{i x}(x, \lambda)=\frac{\partial}{\partial x} f_{i}(x, \lambda)$. 
as $\lambda$ increases. In no sub-interval of $(X)$ are $K$ and $G$ simultaneously independent of $\lambda$ and in no sub-interval of $(X)$ is $G$ identically zero.

IV. Either $\beta_{i} \equiv 0$ for all values of $x$ and $\lambda$ in $(X, \Lambda)$; or else $\beta_{i} \neq 0$ in $a<x<b$ for all $\lambda$ 's in $(\Lambda)$ and one of the following is true,

either (a) $\beta_{i}(a) \equiv 0$ for all $\lambda$ 's in $(\Lambda), \beta_{i}(b) \neq 0,-\alpha_{i}(b) / \beta_{i}(b)$

decreases (or does not increase) as $\lambda$ increases;

$$
\text { or (b) } \beta_{i}(b) \equiv 0, \beta_{i}(a) \neq 0 \text { for all } \lambda \text { 's in }(\Lambda), \alpha_{i}(a) / \beta_{i}(a)
$$

decreases (or does not increase) as $\lambda$ increases;

$$
\text { or (c) } \beta_{i}(a) \neq 0, \beta_{i}(b) \neq 0, \alpha_{i}(a) / \beta_{i}(a) \text { and }-\alpha_{i}(b) / \beta_{i}(b)
$$

decrease (or do not increase) as $\lambda$ increases.

$$
\begin{aligned}
& \text { V.* } \\
& \lim _{\lambda \rightarrow S_{1}}-\frac{\min G}{\min K}=-\infty, \\
& \lim _{\lambda \rightarrow S_{2}}-\frac{\max G}{\max K_{1}}=+\infty .
\end{aligned}
$$

\section{The Sturmin system}

Concerning the system

$$
\begin{gathered}
\frac{d}{d x}\left[K(x, \lambda) \frac{d u}{d x}\right]-G(x, \lambda) u=0, \\
L_{1}[u(a, \lambda)]=0, \quad L_{1}[u(b, \lambda)]=0,
\end{gathered}
$$

STURM's oscillation THEOREM $\dagger$ may be stated with Bôcher $\ddagger$ substantially as follows:

The system (3) satisfying conditions $I-V$ has an infinite set of characteristic numbers such that

$$
\mathscr{L}_{1}<\lambda_{0}<\lambda_{1}<\lambda_{2}<\cdots<\mathscr{L}_{2}
$$

and $U\left(x, \lambda_{p}\right)$, the pth characteristic function, vanishes exactly $p$ times on $a<x<b$.

We seek to determine the number of oscillations of $L_{1}\left[U\left(x, \lambda_{p}\right)\right]$. We notice first that if $\beta_{1} \equiv 0$ for all values of $x$ and $\lambda$ in $(X, \Lambda)$, then all the zeros of $U\left(x, \lambda_{p}\right)$ and $L_{1}\left[U\left(x, \lambda_{p}\right)\right]$ coincide, since $\alpha_{1}(x, \lambda) \neq 0$. Hence we have for $\beta_{1} \equiv 0$ precisely $p$ zeros of $L_{1}\left[U\left(x, \lambda_{p}\right)\right]$ on $a<x<b$.

With Bôcher§ we define

$$
\left\{\alpha_{1} \beta_{1}\right\}=\beta_{1} \alpha_{1 x}-\alpha_{1} \beta_{1 x}+\frac{\alpha_{1}^{2}}{K}-\beta_{1}^{2} G .
$$

\footnotetext{
* This condition may be replaced by other sets of conditions. See Bôcher, Leçons sur les Méthodes de Sturm (hereafter referred to as Leçons) (1917), chap. III, paragraphs 13-15.

†Journal de Mathématiques pureset appliquées, vol. 1 (1836), p. $106 \mathrm{ff}$.

$\ddagger$ Leçons, p. $63 \mathrm{ff}$.

$\$$ Leçons, p. 45.
} 
Let $\beta_{1} \neq 0$ in $(X, \Lambda)$, and consider the zeros of $U(x, \lambda)$ and $L_{1}[U(x, \lambda)]$. For $\lambda=\lambda_{0}, L_{1}\left[U\left(a, \lambda_{0}\right)\right]=0$ and $L_{1}\left[U\left(b, \lambda_{0}\right)\right]=0$, but $U\left(x, \lambda_{0}\right)$ does not vanish on $(X)$. Hence $\left\{\alpha_{1} \beta_{1}\right\}_{\lambda=\lambda_{0}}$ must vanish for some value of $x$ in $(X)$, since if $\left\{\alpha_{1} \beta_{1}\right\}_{\lambda=\lambda_{0}}<0$ throughout $(X), L_{1}\left[U\left(x, \lambda_{0}\right)\right]$ could vanish but once in $(X) ;^{*}$ and if $\left\{\alpha_{1} \beta_{1}\right\}_{\lambda-\lambda_{0}}>0$ for every $x$ in $(X), U\left(x, \lambda_{0}\right)$ would vanish once in $(X)$. $\dagger$ If $\left\{\alpha_{1} \beta_{1}\right\}>0$ for $\lambda \geqq \lambda_{1}$ for all values of $x$ in $(X)$, then the zeros of $L_{1}[U(x, \lambda)]$ and $U(x, \lambda)$ separate. $\dagger$ But $U\left(x, \lambda_{p}\right)$ vanishes exactly $p$ times on $a<x<b$. Hence $L_{1}\left[U\left(x, \lambda_{p}\right)\right]$ vanishes $p-1$ times on $a<x<b$ for $p \geqq 1$.

If $\beta_{1}(a, \lambda) \equiv 0$ in $(\Lambda), \beta_{1}(x, \lambda) \neq 0$ for $a<x \leqq b$, then $\alpha(a) \neq 0$ in $(\Lambda)$. Then the zeros of $L_{1}\left[U\left(x, \lambda_{p}\right)\right]$ and $U\left(x, \lambda_{p}\right)$ separate ${ }^{\prime}$ each other on $a<x \leqq b$, provided $\left\{\alpha_{1} \beta_{1}\right\}_{\lambda=\lambda_{p}}>0$. Let $x_{1}$ be the first zero of $U\left(x, \lambda_{p}\right)$ on $a<x \leqq b$. Then

$$
L_{1}\left[U\left(x_{1}, \lambda_{p}\right)\right]=-\beta_{1}\left(x_{1}, \lambda_{p}\right) K\left(x_{1}, \lambda_{p}\right) U_{x}\left(x_{1}, \lambda_{p}\right) .
$$

Now direct computation shows that

$$
L_{1 x}[U(a)]=\left\{\alpha_{1} \beta_{1}\right\}_{x=a} \frac{K(a) U_{x}(a)}{\alpha_{1}(a)} .
$$

But $K(a) U_{x}(a)$ and $K\left(x_{1}, \lambda_{p}\right) U_{x}\left(x_{1}, \lambda_{p}\right)$ have opposite signs, and $\operatorname{sgn} \beta_{1}\left(x_{1}, \lambda_{p}\right)=\operatorname{sgn} \beta_{1}(b)$. Hence

$$
\operatorname{sgn} L_{1}\left[U\left(x_{1}, \lambda_{p}\right)\right]=\operatorname{sgn} L_{1 x}[U(a)] \cdot \operatorname{sgn} \beta_{1}(b) \operatorname{sgn} \alpha_{1}(a) .
$$

Thus $L_{1}\left[U\left(x, \lambda_{p}\right)\right]$ vanishes or does not vanish in $a<x<x_{1}$ according as $\alpha_{1}(a) \beta_{1}(b)$ is negative or is positive respectively. Hence if $\alpha_{1}(a) \beta_{1}(b)>0$. $L_{1}\left[U\left(x, \lambda_{p}\right)\right]$ has $p-1$ zeros on $a<x<b$, and if $\alpha_{1}(a) \beta_{1}(b)<0$, $L_{1}\left[U\left(x, \lambda_{p}\right)\right]$ has $p$ zeros on $a<x<b$, for $p \geqq 1$.

If $\beta_{1}(b, \lambda)=0$ in $(\Lambda), \beta_{1}(x, \lambda) \neq 0$ for $a \leqq x<b$, then a similar argument can be made with the following result: if $\alpha_{1}(b) \beta_{1}(a)>0, L_{1}\left[U\left(x, \lambda_{p}\right)\right]$ has $p-1$ zeros on $a<x<b$, and if $\alpha_{1}(b) \beta_{1}(a)<0, L_{1}\left[U\left(x, \lambda_{p}\right)\right]$ has $p$ zeros on $a<x<b$, for $p \geqq 1$. Therefore

OscilLATION THEOREM I. If $U\left(x, \lambda_{p}\right)$ is the pth characteristic function of the system (3) satisfying conditions $I-V$ and if $\left\{\alpha_{1} \beta_{1}\right\}>0$ for $\lambda \geqq \lambda_{1}$ for every $x$ in $(X)$, then $L_{1}\left[U\left(x, \lambda_{p}\right)\right]$ will vanish on $a<x<b$ for $p \geqq 1$,

$p$ times if

either $\beta_{1}=0$ in $(X, \Lambda)$;

or $\beta_{1}(a)=0$ in $(\Lambda), \beta_{1}(x, \lambda) \neq 0$ in $a<x \leqq b$ and $\alpha_{1}(a) \beta_{1}(b)<0$;

or $\beta_{1}(b)=0$ in $(\Lambda), \beta_{1}(x, \lambda) \neq 0$ in $a \leqq x<b$ and $\alpha_{1}(b) \beta_{1}(a)<0$;

$p-1$ times if

* Bocher, Leçons, p. 51.

† Ibid., p. $\mathbf{5 0 .}$

$\ddagger$ sgn $f=$ sign of $f$. 
either $\beta_{1} \neq 0$ in $(X, \Lambda)$;

or $\beta_{1}(a) \equiv 0$ in $(\Lambda), \beta_{1}(x, \lambda) \neq 0$ in $a<x \leqq b$ and $\alpha_{1}(a) \beta_{1}(b)>0$;

or $\beta_{1}(b) \equiv 0$ in $(\Lambda), \beta_{1}(x, \lambda) \neq 0$ in $a \leqq x<b$ and $\alpha_{1}(b) \beta_{1}(a)>0$.

Let $L[u(x, \lambda)]=\alpha(x, \lambda) u(x, \lambda)-\beta(x, \lambda) K(x, \lambda) u_{x}(x, \lambda)$, where $\alpha, \beta, \alpha_{x}, \beta_{x}$ are continuous in $(X, \Lambda)$. Then we may state

Oscillation THEOREM II. If $U\left(x, \lambda_{p}\right)$ is the pth characteristic function of (3) satisfying conditions $I-V$, where $\left\{\alpha_{1} \beta_{1}\right\}>0,\{\alpha \beta\}>0$ for $\lambda \geqq \lambda_{1}$ and $\left(\alpha_{1} \beta-\alpha \beta_{1}\right) \neq 0$ in $(X, \Lambda)$, then $L\left[U\left(x, \lambda_{p}\right)\right]$ will vanish on $a<x<b$, for $p \geqq 1$,

$p$ times if

either $\beta_{1} \neq 0$ in $(X, \Lambda)$;

or $\beta_{1}(a) \equiv 0$ in $(\Lambda), \beta_{1}(x, \lambda) \neq 0$ in $a<x \leqq b$ and $\alpha_{1}(a) \beta_{1}(b)>0$;

or $\beta_{1}(b) \equiv 0$ in $(\Lambda), \beta_{1}(x, \lambda) \neq 0$ in $a \leqq x<b$ and $\alpha_{1}(b) \beta_{1}(a)>0$;

$p+1$ times if

either $\beta_{1} \equiv 0$ in $(X, \Lambda)$;

or $\beta_{1}(a) \equiv 0$ in $(\Lambda), \beta_{1}(x, \lambda) \neq 0$ in $a<x \leqq b$ and $\alpha_{1}(a) \beta_{1}(b)<0$;

or $\beta_{1}(b) \equiv 0$ in $(\Lambda), \beta_{1}(x, \lambda) \neq 0$ in $a \leqq x<b$ and $\alpha_{1}(b) \beta_{1}(a)<0$.

Proof: The zeros of $L_{1}\left[U\left(x, \lambda_{p}\right)\right]$ and $L\left[U\left(x, \lambda_{p}\right)\right]$ separate one another on $(X) .^{*}$ But $L_{1}\left[U\left(a, \lambda_{p}\right)\right]=0$ and $L_{1}\left[U\left(b, \lambda_{p}\right)\right]=0$. Hence $L\left[U\left(x, \lambda_{p}\right)\right]$ has one more zero or $a<x<b$, for $p \geqq 1$, than $L_{1}\left[U\left(x, \lambda_{p}\right)\right]$. This proves Theorem II.

\section{The General SELF-ADJOINT SYSTEM}

Consider the system

$$
\begin{gathered}
\frac{d}{d x}\left[K(x, \lambda) \frac{d u}{d x}\right]-G(x, \lambda) u=0, \\
L_{1}[u(a, \lambda)]=L_{1}[u(b, \lambda)], \quad L_{2}[u(a, \lambda)]=L_{2}[u(b, \lambda)],
\end{gathered}
$$

where $L_{1}$ and $L_{2}$ are defined as in (2) and $\beta_{2} \neq 0 \dagger$ in $(X, \Lambda)$. We impose further conditions:

VI.

$$
\left\{\alpha_{i} \beta_{i}\right\} \neq 0 \text { for } \lambda \geqq \lambda_{1},
$$

VII.

$$
\alpha_{1} \beta_{2}-\alpha_{2} \beta_{1} \equiv-1 \text { in }(X, \Lambda) \text {, }
$$

VIII.

$$
\left|\begin{array}{rrrr}
\alpha_{1}(a) & \beta_{1}(a) & \alpha_{1}(b) & \beta_{1}(b) \\
\alpha_{2}(a) & \beta_{2}(a) & \alpha_{2}(b) & \beta_{2}(b) \\
\Delta a_{1}(a) & \Delta \beta_{1}(a) & \Delta \alpha_{1}(b) & \Delta \beta_{1}(b) \\
\Delta \alpha_{2}(a) & \Delta \beta_{2}(a) & \Delta \alpha_{2}(b) & \Delta \beta_{2}(b)
\end{array}\right| \geqq 0 .
$$

* Bócher, Leçons, p. 50.

$\dagger$ This restriction does not involve a loss of generality, since if $\beta_{1}+0, \beta_{2}=0$ then we may interchange $L_{1}$ and $L_{2}$.

$\ddagger \Delta f=f(\lambda+\Delta \lambda)-f(\lambda)$ for $\Delta \lambda>0$. 
In a recent paper* the writer proved the following theorem concerning the system (4), satisfying conditions I-VIII:

There exists one and only one characteristic number of (4) between every pair of characteristic numbers of the Sturmian system (3). If $\lambda_{p}$ represents the ordered characteristic numbers of (3) and $l_{p}$ those of (4) (account being taken of their multiplicity) then

Case I. $l_{p}$ is in the interval $\left(\lambda_{p}, \lambda_{p+1}\right)$ if $L_{2}\left[U\left(b, \lambda_{0}\right)\right] \cdot \phi\left(\mathcal{L}_{1}+\epsilon\right)>0 . \dagger$

Case II. $l_{p}$ is in the interval $\left(\lambda_{p-1}, \lambda_{p}\right), p \geqq 1$, if

$$
L_{2}\left[U\left(b, \lambda_{0}\right)\right] \cdot \phi\left(\mathcal{L}_{1}+\epsilon\right)<0 .
$$

Let $u_{p}(x)=u\left(x, l_{p}\right)$ be the $p$ th characteristic function of (4). We proceed to consider the number of oscillations of $L_{1}\left[u_{p}(x)\right]$. We notice first that if $\lambda=l_{p}$ is a double characteristic number, then $l_{p}$ coincides with $\lambda_{p-1}$, $\lambda_{p}$, or $\lambda_{p+1}$, and $L_{1}\left[u_{p}(x)\right]$ will have the number of oscillations designated by Theorem I.

If $\lambda=l_{p}$ is a simple value, we may discriminate between Case I and Case II, following a method due to Birkhoff. $\ddagger$ If $\beta_{1} \equiv 0$ in $(X, \Lambda)$, the sign of $L_{2}\left[U\left(b, \lambda_{0}\right)\right]$ is the same as $-\alpha_{1}(a) \alpha_{1}(b)$, which is negative, since $\alpha_{1} \neq 0$ in $(X, \Lambda)$. Hence $L_{2}\left[U\left(b, \lambda_{0}\right)\right]$ is negative. Also $\phi\left(\mathcal{L}_{1}+\epsilon\right)$ has the sign of $-\beta_{2}(a) \beta_{2}(b)$, but $\beta_{2} \neq 0$ in $(X, \Lambda)$. Hence $\phi\left(\mathcal{L}_{1}+\epsilon\right)$ is negative, and we have Case I where $l_{p}$ is on the interval $\lambda_{p}<\lambda<\lambda_{p+1}$. By Theorem $I, L_{1}\left[U\left(x, \lambda_{p}\right)\right]$ vanishes exactly $p$ times on $a<x<b$ for $p \geqq 1$, and $L_{1}\left[U\left(x, \lambda_{p+1}\right)\right]$ vanishes $p+1$ times on $a<\dot{x}<b$ for $p \geqq 1$. But $L_{1}[U(b, \lambda)] \neq 0$ for $\lambda_{p}<\lambda<\lambda_{p+1}$. Hence $L_{1}[U(x, \lambda)]$ vanishes $p+1$ times on $a<x<b$ for $\lambda_{p}<\lambda<\lambda_{p+1}$. But the roots of $L_{1}[U(x, \lambda)]$ and $L_{1}[u(x, \lambda)] \S$ separate one another, and $L_{1}[U(a, \lambda)]=0$. Hence $L_{1}[u(x, \lambda)]$ vanishes $p+1$ or $p+2$ times on $a<x<b$ for $\lambda_{p}<\lambda<\lambda_{p+1}$, $p \geqq 1$. Hence $L_{1}\left[u_{p}(x)\right]$ vanishes either $p+1$ or $p+2$ times on $a<x<b$, $p \geqq 1$. But from (4) the number of zeros of $L_{1}\left[u_{p}(x)\right]$ is always even. Therefore we have $p+2$ roots if $p$ is even and $p+1$ roots for $p$ odd, $p \geqq 1$.

If $\beta_{1}(a) \equiv 0$ in $(\Lambda)$ and $\beta_{1}(b) \neq 0$, the sign of $L_{2}\left[U\left(b, \lambda_{0}\right)\right]$ is that of $\alpha_{1}(a) / \beta_{1}(b)$, and $\phi\left(\mathcal{L}_{1}+\epsilon\right)$ has the sign of $\beta_{1}(b) / \alpha_{1}(a)$. Hence we have Case I where $l_{p}$ is on the interval $\lambda_{p}<\lambda<\lambda_{p+1}$. If $\alpha_{1}(a) \beta_{1}(b)<0$, by Theorem I, $L_{1}\left[U\left(x, \lambda_{p}\right)\right]$ vanishes $p$ times on $a<x<b$ and $L_{1}\left[U\left(x, \lambda_{p+1}\right)\right]$ vanishes $p+1$ times on $a<x<b$. Reasoning exactly as above, we find that $L_{1}\left[u_{p}(x)\right]$ vanishes $p+2$ times on $a<x<b$ if $p$ is even and $p+1$

- Existence Theorems for the General, Real, Self-Adjoint Linear System of the Second Order, these Transaction s, vol. 19 (1918), p. 94.

$\dagger \phi(\lambda)=0$ is the characteristic equation of (4) whose roots are the characteristic numbers, $l_{p} . \quad L_{1}+\epsilon$ is a value of $\lambda$ in $(\Lambda)$ near $L_{1}$.

$\ddagger$ Existence and Oscillation Theorem for a Certain Boundary Value Problem, these T r a n sa c t i o n s, vol. 10 (1909), pp. 259-270.

§ Bôcher, Leçons, p. 48. 
times if $p$ is odd, $p \geqq 1$. If $\alpha_{1}(a) \beta_{1}(b)>0$, by Theorem I, $L_{1}\left[U\left(x, \lambda_{p}\right)\right]$ vanishes $p-1$ times on $a<x<b$, and $L_{1}\left[U\left(x, \lambda_{p+1}\right)\right]$ vanishes $p$ times on $a<x<b$. But $L_{1}[U(b, \lambda)] \neq 0$ for $\lambda_{p}<\lambda<\lambda_{p+1}$, and $L_{1}[U(a, \lambda)]=0$. The roots of $L_{1}[U(x, \lambda)]$ and $L_{1}[u(x, \lambda)]$ separate one another. Hence $L_{1}\left[u_{p}(x)\right]$ vanishes either $p$ or $p+1$ times on $a<x<b, p \geqq 1$. But from (4) the number of zeros of $L_{1}\left[u_{p}(x)\right]$ is always even. Therefore we have $p$ roots if $p$ is even and $p+1$ roots if $p$ is odd.

If $\beta_{1}(b) \equiv 0$ in $(\Lambda)$ and $\beta_{1}(a) \neq 0$, the sign of $L_{2}\left[U\left(b, \lambda_{0}\right)\right]$ is that of $-\beta_{1}(a) / \alpha_{1}(b)$, and $\phi\left(\mathcal{L}_{1}+\epsilon\right)$ has the sign of $-\beta_{1}(a) / \alpha_{1}(b)$. Hence we have Case I again. If $\alpha_{1}(b) \beta_{1}(a)<0$ we proceed as before and obtain $p+2$ zeros of $L_{1}\left[u_{p}(x)\right]$ on $a<x<b$ if $p$ is even and $p+1$ zeros if $p$ is odd, $p \geqq 1$. If $\alpha_{1}(b) \beta_{1}(a)>0$, we obtain $p$ zeros of $L_{1}\left[u_{p}(x)\right]$ on $a<x<b$ if $p$ is even and $p+1$ zeros if $p$ is odd, $p \geqq 1$.

If $\beta_{1} \neq 0$ in $(X, \Lambda)$, the sign of $L_{2}\left[U\left(b, \lambda_{0}\right)\right]$ is that of $\beta_{1}(a) / \beta_{1}(b)$, which is positive. The sign of $\phi\left(\mathscr{L}_{1}+\epsilon\right)$ is that of $\beta_{1}(a) \beta_{2}(b)-\beta_{2}(a) \beta_{1}(b)$ if $\beta_{1}(a) \beta_{2}(b)-\beta_{2}(a) \beta_{1}(b)$ does not vanish. If

$$
\beta_{1}(a) \beta_{2}(b)-\beta_{2}(a) \beta_{1}(b)=0,
$$

the sign of $\phi\left(\mathcal{L}_{1}+\epsilon\right)$ is that of $\beta_{1}(a) \beta_{1}(b)$, which is positive. Accordingly, if $\beta_{1}(a) \beta_{2}(b)-\beta_{2}(a) \beta_{1}(b) \geqq 0$, we have Case $\mathrm{I}$, and $l_{p}$ is on $\lambda_{p}<\lambda<\lambda_{p+1}$. By Theorem I, $L_{1}\left[U\left(x, \lambda_{p}\right)\right]$ vanishes $p-1$ times, and, proceeding as above, we find that $L_{1}\left[u_{p}(x)\right]$ has $p$ zeros if $p$ is even and $p+1$ zeros if $p$ is odd, $p \geqq 1$. Likewise, if $\beta_{1}(a) \beta_{2}(b)-\beta_{2}(a) \beta_{1}(b)<0, l_{p}$ is on $\lambda_{p-1}<\lambda<\lambda_{p}$, and $L\left[i_{p}(x)\right]$ will vanish $p$ times if $p$, is even and $p-1$ times if $p$ is odd, $p \geqq 1$.

Summarizing the various kinds of coefficients of the boundary conditions of (4) as follows:

$A: \quad \beta_{1} \equiv 0$ in $(X, \Lambda)$;

$B^{-}:$Either $\beta_{1}(a) \equiv 0$ in $(\Lambda), \beta_{1}(x, \lambda) \neq 0$ in $a<x \leqq b$, and

or

$$
\beta_{1}(b) \equiv 0 \text { in }(\Lambda), \beta_{1}(x, \lambda) \neq 0 \text { in } a \leqq x<b \text {, and } \alpha_{1}(a)<0 ;
$$

$$
\alpha_{1}(b) \beta_{1}(a)<0 ;
$$

$B^{+}:$Either $\beta_{1}(a) \equiv 0$ in $(\Lambda), \beta_{1}(x, \lambda) \neq 0$ in $a<x \leqq b$, and

$$
\text { or } \quad \beta_{1}(b) \equiv 0 \text { in }(\Lambda), \beta_{1}(x, \lambda) \neq 0 \text { in } a \leqq x<b \text {, and }
$$

$$
\alpha_{1}(a) \beta_{1}(b)>0 ;
$$

$$
\alpha_{1}(b) \beta_{1}(a)>0 ;
$$

$C^{+}: \beta_{1} \neq 0$ in $(X, \Lambda)$ and $\beta_{1}(a) \beta_{2}(b)-\beta_{2}(a) \beta_{1}(b) \geqq 0$ in $(\Lambda)$;

$C^{-}: \beta_{1} \neq 0$ in $(X, \Lambda)$ and $\beta_{1}(a) \beta_{2}(b)-\beta_{2}(a) \beta_{1}(b)<0$ in $(\Lambda)$;

we state

Oscillatiox THEOREM III. If $u_{p}(x)$ is the pth characteristic function corresponding to a simple value of the system (4) satisfying conditions I-VIII, 
then the number of zeros of $L_{1}\left[u_{p}(x)\right]$ on $a<x<b$ for $p \geqq 1$ is given by the following table:

\begin{tabular}{|c|c|c|}
\hline \multirow[t]{2}{*}{ Case } & \multicolumn{2}{|c|}{ Number of zeros } \\
\hline & $p=2 m$ & $p=2 m+1$ \\
\hline$A$ or $B^{-}$ & $p+2$ & $p+1$ \\
\hline $\begin{array}{c}B^{+} \text {or } C^{-} \\
C^{+}\end{array}$ & $\begin{array}{l}p \\
p\end{array}$ & $\begin{array}{l}p+1 \\
p-1\end{array}$ \\
\hline
\end{tabular}

Furthermore, we notice that, since $\alpha_{1} \beta_{2}-\alpha_{2} \beta_{1} \neq 0$ in $(X, \Lambda)$, the zeros of $L_{1}\left[u_{p}(x)\right]$ and $L_{2}\left[u_{p}(x)\right]$ separate one another on $X,^{*}$ and, if $\lambda=l_{p}$ is a double value, the number of zeros of $L_{2}\left[u_{p}(x)\right]$ is given by Theorem II. If $\lambda=l_{p}$ is a simple value, both $L_{1}\left[u_{p}(x)\right]$ and $L_{2}\left[u_{p}(x)\right]$ by (4) have an even number of zeros on $(X)$. Hence they oscillate the same number of times. Therefore

COROLlaRY. The number of zeros of $L_{2}\left[u_{p}(x)\right]$ on $a<x<b$, for $p \geqq 1$, is precisely the number given in the table of Theorem III.

From the foregoing results we may deduce the number of oscillations of $u_{p}(x)$. If $\lambda=l_{p}$ is a double value, then $u_{p}(x)$ will differ from $U\left(x, \lambda_{p-1}\right)$, $U\left(x, \lambda_{p}\right)$, or $U\left(x, \lambda_{p+1}\right)$ by at most a non-vanishing factor, and the number of zeros of $u_{p}(x)$ will be given by the Sturmian Oscillation Theorem.

For a simple value we consider the following cases:

If $\beta_{1} \equiv 0$ in $(X, \Lambda)$ the zeros of $u_{p}(x)$ and $L_{1}\left[u_{p}(x)\right]$ coincide. Hence $u_{p}(x)$ has $p+2$ zeros if $p=2 m$ and $p+1$ zeros if $p=2 m+1$.

If $\beta_{1} \neq 0$ in $(X, \Lambda)$ or $\beta_{1}(a) \equiv 0, \beta_{1}(b) \neq 0$ or $\beta_{1}(b) \equiv 0, \beta_{1}(a) \neq 0$, we write the first boundary condition of (4)

where

$$
u_{p}(a) \cdot P(a)=u_{p}(b) \cdot P(b),
$$

$$
P_{1}(x)=\alpha_{1}(x)-\beta_{1}(x) K(x) u_{p}^{\prime}(x) / u_{p}(x) .
$$

If $P_{1}(a) P_{1}(b)>0, u_{p}(a) u_{p}(b)$ will be positive and $u_{p}(x)$ has an even number of roots. But the zeros of $u_{p}(x)$ and $L_{1}\left[u_{p}(x)\right]$ separate. Hence $u_{p}(x)$ and $L_{1}\left[u_{p}(x)\right]$ have the same number of zeros on $a<x<b$. If $P_{1}(a) P_{1}(b)<0, u_{p}(a) u_{p}(b)$ will be negative and $u_{p}(x)$ has an odd number of zeros. Hence $u_{p}(x)$ will have one more or one less zero than $L_{1}\left[u_{p}(x)\right]$ on $a<x<b$.

It is also to be noticed that in Case I, since $l_{p}$ is on $\left(\lambda_{p}, \lambda_{p+1}\right), u_{p}(x)$ can vanish not more than $p+2$ times nor less than $p-1$ times. In Case II, since $l_{p}$ is on $\left(\lambda_{p-1}, \lambda_{p}\right), u_{p}(x)$ can vanish not more than $p+1$ times nor less than $p-2$ times. $\dagger$

Designating the condition $P_{1}(a) \cdot P_{1}(b)>0$ by $P^{+}$and the condition $P_{1}(a) \cdot P_{1}(b)<0$ by $P^{-}$, we may state

* Bocher, Leçons, p. 50.

† Cf. Cor., p. 96 of the paper of the author referred to above. 
OSCILLATION THEOREM IV. If $u_{p}(x)$ is the pth characteristic function corresponding to a simple value of the system (4) satisfying conditions I-VIII, then the number of zeros of $u_{p}(x)$ on $a<x<b$, for $p \geqq 1$, is given by the following table:

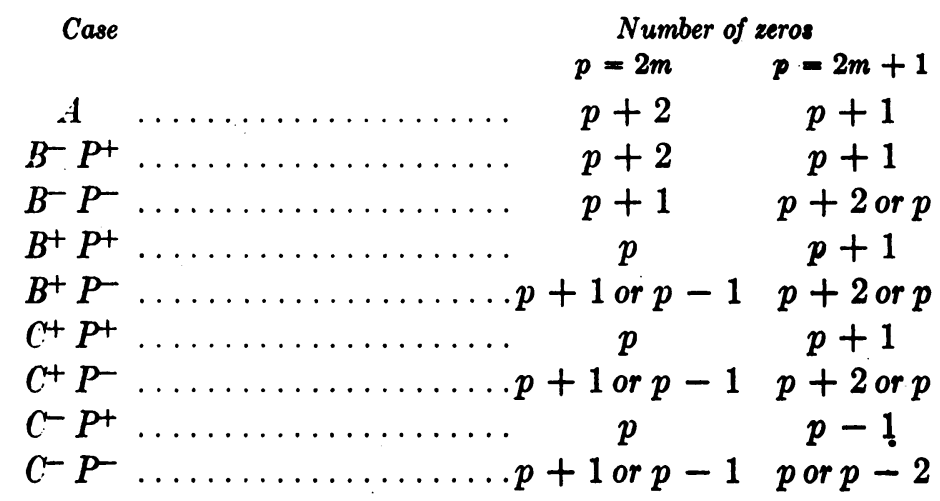

Note: if in particular we choose

$$
\begin{aligned}
& \bar{\alpha}_{i}(x, \lambda)=\frac{(b-x) \alpha_{i}(\lambda)+(x-a) \gamma_{i}(\lambda)}{b-a} \\
& \bar{\beta}_{i}(x, \lambda)=\frac{(b-x) \beta_{i}(\lambda)+(a-x) \delta_{i}(\lambda)}{b-a}
\end{aligned}
$$

the system (4) becomes identical with that of (4) of the paper by the writer to which reference has been made above. If $\beta_{i} \delta_{i}>0$, it will be necessary to modify the boundary conditions of (4) by taking

$$
L_{i}[u(a, \lambda)]=-L_{i}[u(b, \lambda)]
$$

with condition VII replaced by

$$
\alpha_{1}(a) \beta_{2}(a)-\alpha_{2}(a) \beta_{1}(a)=\alpha_{2}(b) \beta_{1}(b)-\alpha_{1}(b) \beta_{2}(b)=-1,
$$

if only one of the boundary conditions is modified. If both boundary conditions are modified, condition VII remains unchanged. In either case conditions I-VI and VIII will be satisfied. The oscillation theorems will be true with the modification that $L_{i}\left[u_{p}(x)\right]$ will have an odd number of zeros.

UNIVERSITY OF TExag, AUstin, Texis 